MATHEMATICS OF COMPUTATION

Volume 70, Number 233, Pages 281-296

S $0025-5718(00) 01260-6$

Article electronically published on June 12, 2000

\title{
ERROR BOUNDS \\ FOR INTERPOLATORY QUADRATURE RULES ON THE UNIT CIRCLE
}

\author{
J. C. SANTOS-LEÓN
}

\begin{abstract}
For the construction of an interpolatory integration rule on the unit circle $T$ with $n$ nodes by means of the Laurent polynomials as basis functions for the approximation, we have at our disposal two nonnegative integers $p_{n}$ and $q_{n}, p_{n}+q_{n}=n-1$, which determine the subspace of basis functions. The quadrature rule will integrate correctly any function from this subspace. In this paper upper bounds for the remainder term of interpolatory integration rules on $T$ are obtained. These bounds apply to analytic functions up to a finite number of isolated poles outside $T$. In addition, if the integrand function has no poles in the closed unit disc or is a rational function with poles outside $T$, we propose a simple rule to determine the value of $p_{n}$ and hence $q_{n}$ in order to minimize the quadrature error term. Several numerical examples are given to illustrate the theoretical results.
\end{abstract}

\section{INTRODUCTION}

This paper deals with the numerical calculation of integrals around the unit circle in the complex plane, that is, integrals of the form

$$
I(f)=\int_{-\pi}^{\pi} f\left(e^{i \theta}\right) d \psi(\theta),
$$

where $\psi$ is a distribution function (real valued, bounded and nondecreasing ) on $(-\pi, \pi)$. We write $T=\{z \in \mathbb{C}:|z|=1\}$ for the unit circle.

Jones, Njåstad and Thron in [6] studied the so-called Szegö quadrature formulas for the estimations of integrals (1). They are similar to the Gaussian formulas on the real line, but the role played by polynomials and orthogonal polynomials is now played by Laurent polynomials and para-orthogonal polynomials. These topics are described below.

Let $(p, q)$ be a pair of integers where $p \leq q$. We denote by $\Lambda_{p, q}$ the linear space of all functions of the form $\sum_{j=p}^{q} c_{j} z^{j}, c_{j} \in \mathbb{C}$. The functions of $\Lambda_{p, q}$ are called Laurent polynomials or L-polynomials. We write $\Lambda$ for the linear space of all L-polynomials.

Received by the editor February 2, 1999.

2000 Mathematics Subject Classification. Primary 41A55, 65D30.

Key words and phrases. Error bounds, quadrature formulas, singular integrands, Szegö polynomials.

This work was supported by the Ministry of Education and Culture of Spain under contract PB96-1029.

(C)2000 American Mathematical Society 
Consider the inner product on $\Lambda \times \Lambda$ given by

$$
(f, g)=\int_{-\pi}^{\pi} f\left(e^{i \theta}\right) \overline{g\left(e^{i \theta}\right)} d \psi(\theta) .
$$

Let $\left\{\rho_{n}\right\}_{0}^{\infty}$ be the sequence of polynomials obtained by orthogonalization of $\left\{z^{n}\right\}_{0}^{\infty}$ with respect to the inner product (2). The sequence $\left\{\rho_{n}\right\}_{0}^{\infty}$ is the sequence of Szegö polynomials with respect to the distribution function $\psi$. As is well known (see, e.g., Theorem 3.4 in [5]) $\rho_{n}$ has its zeros in the region $|z|<1$. Thus they are not adequate as nodes for quadrature formulas to estimate integrals (11) on the unit circle.

Theorem 1 ([6]). Let $\left\{\rho_{n}\right\}_{0}^{\infty}$ be the sequence of Szegö polynomials with respect to the distribution function $\psi$. Let $\left\{\kappa_{n}\right\}_{0}^{\infty}$ be a sequence of complex numbers satisfying $\left|\kappa_{n}\right|=1, n \geq 0$. Let $B_{n}\left(z, \kappa_{n}\right)=\rho_{n}(z)+\kappa_{n} \rho_{n}^{*}(z)$, where $\rho_{n}^{*}(z)=z^{n} \bar{\rho}_{n}(1 / z)$. Then $B_{n}\left(z, \kappa_{n}\right)$ has $n$ distinct zeros $\zeta_{m}^{(n)}\left(\kappa_{n}\right)$ located on $T$. Let

$$
\lambda_{m}^{(n)}\left(\kappa_{n}\right)=\int_{-\pi}^{\pi} \frac{B_{n}\left(z, \kappa_{n}\right)}{\left(z-\zeta_{m}^{(n)}\left(\kappa_{n}\right)\right) B_{n}^{\prime}\left(\zeta_{m}^{(n)}\left(\kappa_{n}\right), \kappa_{n}\right)} d \psi(\theta), 1 \leq m \leq n .
$$

Then

$$
I(f)=\int_{-\pi}^{\pi} f\left(e^{i \theta}\right) d \psi(\theta)=\sum_{m=1}^{n} \lambda_{m}^{(n)}\left(\kappa_{n}\right) f\left(\zeta_{m}^{(n)}\left(\kappa_{n}\right)\right)
$$

for all $f \in \Lambda_{-(n-1), n-1}$. It holds that $\lambda_{m}^{(n)}\left(\kappa_{n}\right)>0,1 \leq m \leq n$, and the quadrature formula (3) gives the largest domain of validity, that is, there cannot exist an n-point quadrature formula $\mu(f)=\sum_{m=1}^{n} \lambda_{m} f\left(\alpha_{m}\right), \alpha_{m} \in T$, which correctly integrates any function $f \in \Lambda_{-(n-1), n}$ or any function $f \in \Lambda_{-n, n-1}$.

The polynomials $B_{n}\left(z, \kappa_{n}\right), n \geq 0$, are the para-orthogonal polynomials with respect to the distribution function $\psi$.

In 2] Bultheel, González-Vera, Hendriksen and Njåstad proved that the Szegö quadrature process converges as $n$ tends to infinity to $I(f)$, for all integrable functions $f$ on $T$ with respect to the measure $d \psi$. They also introduced the so-called interpolatory rules on the unit circle.

Definition 1. 2] Let $x_{j}, 1 \leq j \leq n$, be $n$ distinct given points on $T$. Let $p_{n}$ and $q_{n}$ be nonnegative integers such that $p_{n}+q_{n}=n-1$. A quadrature formula $I_{n}(f)=\sum_{j=1}^{n} \mu_{j} f\left(x_{j}\right), \quad \mu_{j} \in \mathbb{C}$, to approximate the integral (10) is said to be of interpolatory type in $\Lambda_{-p_{n}, q_{n}}$ if $\mu_{j}=\int_{-\pi}^{\pi} L_{j}\left(e^{i \theta}\right) d \psi(\theta)$, where $L_{j}(z)=$ $x_{j}^{p_{n}} N(z) /\left(z^{p_{n}}\left(z-x_{j}\right) N^{\prime}\left(x_{j}\right)\right), \quad 1 \leq j \leq n$, are the fundamental Lagrange L-polynomials in $\Lambda_{-p_{n}, q_{n}}$, with respect to the nodes $x_{j}, 1 \leq j \leq n$, and $N(z)=$ $\Pi_{j=1}^{n}\left(z-x_{j}\right)$.

Thus $I_{n}(f)=I(L)$, where $L(z)=\sum_{j=1}^{n} f\left(x_{j}\right) L_{j}(z)$ is the L-polynomial in $\Lambda_{-p_{n}, q_{n}}$ interpolating $f$ at $x_{j}, 1 \leq j \leq n$. An $n$-point Szegö quadrature formula is of interpolatory type ([2]) in $\Lambda_{-p_{n}, q_{n}}$ for any $p_{n}$ and $q_{n}$ nonnegative integers satisfying $p_{n}+q_{n}=n-1$. The following theorem is proved in [1] for the general case where the basis functions for the approximation is the set of rational functions with prescribed poles, which includes the Laurent polynomials as a particular case. We state it here for the Laurent polynomials. 
Theorem 2. Assume we are interested in the estimation of integrals of the form $I_{\rho}(f)=\int_{-\pi}^{\pi} f\left(e^{i \theta}\right) \rho(\theta) d \theta$, where $\rho(\theta)$ is a complex valued function such that $\int_{-\pi}^{\pi}|\rho(\theta)| d \theta<\infty$. Let $\omega(\theta)$ be a nonnegative weight function such that $\int_{-\pi}^{\pi}|\rho(\theta)|^{2} / \omega(\theta) d \theta<\infty$. Let $\left\{x_{j, n}\right\}_{j=1}^{n}, n \geq 1$, be the zeros of the para-orthogonal polynomial of degree $n$ with respect to the distribution function $s(\theta)=\int_{-\pi}^{\theta} \omega(t) d t$. Let $\sum_{j=1}^{n} A_{j, n} f\left(x_{j, n}\right)$ be the quadrature formula of interpolatory type in $\Lambda_{-p_{n}, q_{n}}$ with nodes $\left\{x_{j, n}\right\}_{j=1}^{n}$ to approximate the integral $I_{\rho}(f)$. As usual, $p_{n}$ and $q_{n}$ are nonnegative integers satisfying $p_{n}+q_{n}=n-1$. If $\lim _{n \rightarrow \infty} p_{n}=\lim _{n \rightarrow \infty} q_{n}=\infty$, then

$$
\lim _{n \rightarrow \infty} \sum_{j=1}^{n} A_{j, n} f\left(x_{j, n}\right)=I_{\rho}(f)
$$

for all functions $f$ bounded on $T$ for which $I_{\rho}(f)$ exists as a Riemann integral.

In this paper it is assumed that the numerical calculation of integrals of the form (11) is done by means of quadrature formulas $I_{n}(f)$ of interpolatory type in $\Lambda_{-p_{n}, q_{n}}$, where $p_{n}$ and $q_{n}$ are nonnegative integers satisfying $p_{n}+q_{n}=n-1$. We will also assume that the remainder term

$$
R_{n}(f)=I(f)-I_{n}(f)
$$

satisfies

$$
\left|R_{n}\left(z^{k}\right)\right| \leq M_{n}, k \leq-\left(p_{n}+1\right), k \geq q_{n}+1, k \in \mathbb{Z}, n \geq 1,
$$

where $M_{n}>0$ is a constant independent of $k$. By construction, $R_{n}\left(z^{k}\right)=0,-p_{n} \leq$ $k \leq q_{n}$. Condition (4) is satisfied by Szegö quadrature formulas (3). Indeed, let the moments $m_{k}$ be given by

$$
m_{k}=I\left(z^{k}\right), k \in \mathbb{Z} .
$$

Then, taking into account that $\left|m_{k}\right| \leq m_{0}, k \in \mathbb{Z}$, and the coefficients $\lambda_{m}^{(n)}\left(\kappa_{n}\right)$ of the Szegö quadrature formula are positive, we get

$$
\left|R_{n}\left(z^{k}\right)\right|=\left|m_{k}-I_{n}\left(z^{k}\right)\right| \leq m_{0}+\sum_{m=1}^{n} \lambda_{m}^{(n)}\left(\kappa_{n}\right)\left|\left(\zeta_{m}^{(n)}\left(\kappa_{n}\right)\right)^{k}\right| .
$$

Since the nodes $\zeta_{m}^{(n)}\left(\kappa_{n}\right)$ are located on $T$ and

$$
I_{n}(1)=\sum_{m=1}^{n} \lambda_{m}^{(n)}\left(\kappa_{n}\right)=I(1)=m_{0}, n \geq 1,
$$

it results that

$$
\left|R_{n}\left(z^{k}\right)\right| \leq M_{n}=2 m_{0}, k \in \mathbb{Z} .
$$

Quadrature formulas of interpolatory type as in Theorem 2 that is, with nodes the zeros of para-orthogonal polynomials with respect to a given distribution function, also satisfy (4). This is due to the existence ([1]) of an absolute constant $B>0$ such that $\sum_{j=1}^{n}\left|A_{j, n}\right|<B, n \geq 1$. Indeed,

$$
\left|R_{n}\left(z^{k}\right)\right|=\left|I\left(z^{k}\right)-I_{n}\left(z^{k}\right)\right| \leq m_{0}+\sum_{j=1}^{n}\left|A_{j, n}\right| \leq m_{0}+B, n \geq 1, k \in \mathbb{Z} .
$$


In the following theorem we consider the particular case of the weight function $\omega=1$ in Theorem 2 We deduce that one can take $M_{n}=2 m_{0}$ in (4). We will make use of the well known result that the orthogonal polynomials with respect to the distribution $\psi(\theta)=\int_{-\pi}^{\theta} \omega(t) d t=\theta+\pi$ are given by $([9]) \rho_{n}=z^{n}, n \geq 0$, and hence the para-orthogonal polynomials (see Theorem 1) are given by $B_{n}\left(z, \kappa_{n}\right)=$ $z^{n}+\kappa_{n}, \kappa_{n} \in \mathbb{C},\left|\kappa_{n}\right|=1, n \geq 0$.

Theorem 3. Let $I_{n}(f)=\sum_{j=1}^{n} c_{j, n} f\left(z_{j, n}\right)$ be the quadrature formula to approximate integrals (11) of interpolatory type in $\Lambda_{-p_{n}, q_{n}}$ with uniformly distributed nodes $z_{j, n}$ on $T$, that is, the nodes are the roots of $z^{n}+\kappa_{n}=0, \kappa_{n} \in \mathbb{C},\left|\kappa_{n}\right|=1, n \geq 1$. As usual, $p_{n}$ and $q_{n}$ are nonnegative integers satisfying $p_{n}+q_{n}=n-1$. For $k \in \mathbb{Z}$ one can write $k=r_{k} n+s_{k}, r_{k} \in \mathbb{Z}, 0 \leq s_{k} \leq n-1, n \geq 1$. Then $I_{n}\left(z^{k}\right)=m_{s_{k}} / z_{1, n}^{s_{k}-k}$ if $s_{k} \leq q_{n}$ and $I_{n}\left(z^{k}\right)=m_{s_{k}-n} / z_{1, n}^{s_{k}-n-k}$ otherwise. Hence $\left|R_{n}\left(z^{k}\right)\right| \leq 2 m_{0}, k \in \mathbb{Z}, n \geq 1$.

Proof. We know ([8]),

$$
c_{j, n}=\frac{1}{n} \sum_{\ell=-p_{n}}^{q_{n}} m_{\ell} \frac{w^{(1-j) \ell}}{z_{1, n}^{\ell}}, 1 \leq j \leq n, w=e^{\frac{2 \pi i}{n}}, n \geq 1 .
$$

Since the nodes $z_{j, n}$ can be calculated by means of $z_{j, n}=w^{j-1} z_{1, n}, 1 \leq j \leq n$, we deduce for $k \in \mathbb{Z}$

$I_{n}\left(z^{k}\right)=\frac{1}{n} \sum_{j=1}^{n}\left(\sum_{\ell=-p_{n}}^{q_{n}} m_{\ell} \frac{w^{(1-j) \ell}}{z_{1, n}^{\ell}}\right) w^{(j-1) k} z_{1, n}^{k}=\frac{z_{1, n}^{k}}{n} \sum_{\ell=-p_{n}}^{q_{n}} \frac{m_{\ell}}{z_{1, n}^{\ell}} \sum_{j=1}^{n}\left(w^{k-\ell}\right)^{j-1}$.

We can write $k=r_{k} n+s_{k}, r_{k} \in \mathbb{Z}, 0 \leq s_{k} \leq n-1$. Hence

$$
I_{n}\left(z^{k}\right)=\frac{z_{1, n}^{k}}{n} \sum_{\ell=-p_{n}}^{q_{n}} \frac{m_{\ell}}{z_{1, n}^{\ell}} \sum_{j=1}^{n}\left(w^{s_{k}-\ell}\right)^{j-1} .
$$

Note that $w^{s_{k}-\ell}=1$ if and only if $s_{k}-\ell$ is a multiple of $n$. Since $-n+1 \leq s_{k}-\ell \leq$ $2 n-2$, (take into account $0 \leq s_{k} \leq n-1$ and $-n+1 \leq-p_{n} \leq \ell \leq q_{n} \leq n-1$ ) the value $s_{k}-\ell$ is a multiple of $n$ if and only if $s_{k}-\ell=0$ or $s_{k}-\ell=n$. In these cases it holds $\sum_{j=1}^{n}\left(w^{s_{k}-\ell}\right)^{j-1}=n$. If $s_{k}-\ell \neq 0$ and $s_{k}-\ell \neq n$, then $\sum_{j=1}^{n}\left(w^{s_{k}-\ell}\right)^{j-1}=\frac{1-w^{\left(s_{k}-\ell\right) n}}{1-w^{s_{k}-\ell}}=0$. Furthermore, if $s_{k} \leq q_{n}$, then taking into account that $-q_{n} \leq-\ell \leq p_{n}$, we deduce $-q_{n} \leq s_{k}-\ell \leq p_{n}+q_{n}=n-1$. So, it takes place $s_{k}-\ell=0$ but no $s_{k}-\ell=n$. If $s_{k}>q_{n}$, then it takes place $s_{k}-\ell=n$ but no $s_{k}-\ell=0$. The proof follows.

In this paper we are interested in the calculation of upper bounds for the remainder term $R_{n}(f)$ for interpolatory quadrature rules $I_{n}(f)$ in $\Lambda_{-p_{n}, q_{n}}$ for functions $f$ analytic in a simply connected domain $D$ up to a finite number of isolated poles outside $T$. We will assume that $D$ contains $T$ in its interior. This class of functions is equal to the set of all functions $f$ that can be written in the form

$$
f(z)=\frac{g(z)}{\left(z-\alpha_{1}\right)^{\tau_{1}} \cdots\left(z-\alpha_{\nu}\right)^{\tau_{\nu}}},
$$

where $\tau_{j} \geq 0, \tau_{j} \in \mathbb{N}, \alpha_{j} \in \mathbb{C},\left|\alpha_{j}\right| \neq 1, \alpha_{k} \neq \alpha_{j}, 1 \leq j, k \leq \nu$ and $g$ is analytic in $D$. 
Note that if the interpolatory rules are constructed as in Theorem 2, then they converge for all functions of the form (55). Error bounds for Szegö quadrature formulas of analytic functions were given in 2], and for the particular case of integrals that represent Carathéodory functions and real parts of such integrals in [7]. In 8] error bounds for interpolatory integration rules were studied for analytic functions.

Let $\Gamma$ be a positively oriented Jordan curve in $D$ that contains $T$ in its interior, and that does not pass through any of the singular points $\alpha_{1}, \ldots, \alpha_{\nu}$. By the Cauchy integral formula

$$
g(z)=\frac{1}{2 \pi i} \int_{\Gamma} \frac{g(\zeta)}{\zeta-z} d \zeta, z \in T
$$

From (5) and (6) is straightforward to deduce that

$$
R_{n}(f)=\frac{1}{2 \pi i} \int_{\Gamma} K_{n}(\zeta) g(\zeta) d \zeta
$$

where

$$
K_{n}(\zeta)=R_{n}\left(\frac{1}{(\zeta-z)\left(z-\alpha_{1}\right)^{\tau_{1}} \cdots\left(z-\alpha_{\nu}\right)^{\tau_{\nu}}}\right), \zeta \in \Gamma, z \in T .
$$

Thus from (7) we obtain

$$
\left|R_{n}(f)\right| \leq \frac{\ell(\Gamma)}{2 \pi} \max _{\zeta \in \Gamma}\left|K_{n}(\zeta)\right| \max _{\zeta \in \Gamma}|g(\zeta)|,
$$

where $\ell(\Gamma)$ denotes the length of $\Gamma$.

The structure of the paper is the following. In Section 2 we obtain, by means of equation (9), upper bounds for the remainder term $R_{n}(f)$ for functions $f$ of the form (5). These bounds are studied in Section 3. As a result we propose guidelines which help us in the choice of the parameters $p_{n}$ and $q_{n}$ at our disposal for the construction of the interpolatory quadrature formula in order to minimize the error. In Section 4 we illustrate the proposed guidelines through several numerical examples.

\section{ERROR BOUNDS}

For clearness and in order to show the idea that we will use to bound the error term in the general case of multiple poles, we first consider analytic functions and functions with simple poles.

Theorem 4. Let $I_{n}$ be a quadrature formula of interpolatory type in $\Lambda_{-p_{n}, q_{n}}$ where $p_{n}$ and $q_{n}$ are nonnegative integers satisfying $p_{n}+q_{n}=n-1$. Assume that its corresponding error term satisfies (4). Let $f$ be a function of the form (5) with $\tau_{j}=1,1 \leq j \leq \nu$, and let $\Gamma$ be a positively oriented Jordan curve in $D$ that contains $T$ in its interior and that does not pass through any of the singular points $\alpha_{1}, \ldots, \alpha_{\nu}$. As usual $D$ is a simply connected domain containing $T$ in its interior. Then for $n \geq 1$, it holds that

$$
\begin{aligned}
\left|R_{n}(f)\right| \leq \frac{M_{n} \ell(\Gamma)}{2 \pi}\left[\frac{1}{e b^{q_{n}+1}(b-1)}+\sum_{\left|\alpha_{j}\right|<1} \frac{\left|\alpha_{j}\right|^{p_{n}}}{e_{j}\left|P^{\prime}\left(\alpha_{j}\right)\right|\left(1-\left|\alpha_{j}\right|\right)}\right. \\
\left.+\sum_{\left|\alpha_{j}\right|>1} \frac{1}{e_{j}\left|P^{\prime}\left(\alpha_{j}\right)\right|\left(\left|\alpha_{j}\right|-1\right)\left|\alpha_{j}\right|^{q_{n}+1}}\right] \max _{\zeta \in \Gamma}|g(\zeta)|,
\end{aligned}
$$


where $\ell(\Gamma)$ denotes the length of $\Gamma, M_{n}$ is defined by (44), $P(z)=\left(z-\alpha_{1}\right) \cdots\left(z-\alpha_{\nu}\right)$, $e=\min _{\zeta \in \Gamma}|P(\zeta)|$, and

$$
b=\min _{\zeta \in \Gamma}|\zeta|, e_{j}=\min _{\zeta \in \Gamma}\left|\zeta-\alpha_{j}\right|, 1 \leq j \leq \nu .
$$

Proof. For $\zeta \in \Gamma$ and $z \in T$ we can make the partial fraction decomposition

$$
\frac{1}{(\zeta-z)\left(z-\alpha_{1}\right) \cdots\left(z-\alpha_{\nu}\right)}=\frac{B_{0}}{\zeta-z}+\frac{B_{1}}{z-\alpha_{1}}+\cdots+\frac{B_{\nu}}{z-\alpha_{\nu}},
$$

where

$$
B_{0}=\frac{1}{P(\zeta)}, B_{j}=\frac{1}{\left(\zeta-\alpha_{j}\right) P^{\prime}\left(\alpha_{j}\right)}, 1 \leq j \leq \nu
$$

Thus

$$
\begin{aligned}
K_{n}(\zeta) & =R_{n}\left(\frac{1}{(\zeta-z)\left(z-\alpha_{1}\right) \cdots\left(z-\alpha_{\nu}\right)}\right) \\
& =B_{0} R_{n}\left(\frac{1}{\zeta-z}\right)+\sum_{j=1}^{\nu} B_{j} R_{n}\left(\frac{1}{z-\alpha_{j}}\right), z \in T, \zeta \in \Gamma .
\end{aligned}
$$

Let $\alpha$ be a complex number, $|\alpha|>1$, then taking into account that $z \in T$ and $R_{n}\left(z^{k}\right)=0,-p_{n} \leq k \leq q_{n}, n \geq 1$, we get

$$
\begin{aligned}
R_{n}\left(\frac{1}{z-\alpha}\right) & =R_{n}\left(-\frac{1}{\alpha} \sum_{k \geq 0}\left(\frac{z}{\alpha}\right)^{k}\right) \\
& =-\sum_{k \geq 0} \frac{1}{\alpha^{k+1}} R_{n}\left(z^{k}\right)=-\sum_{k \geq q_{n}+1} \frac{1}{\alpha^{k+1}} R_{n}\left(z^{k}\right) .
\end{aligned}
$$

Thereby from (4) one can deduce

$$
\left|R_{n}\left(\frac{1}{z-\alpha}\right)\right| \leq M_{n} \sum_{k \geq q_{n}+1}\left(\frac{1}{|\alpha|}\right)^{k+1}=\frac{M_{n}}{|\alpha|^{q_{n}+1}(|\alpha|-1)}, z \in T,|\alpha|>1 .
$$

In particular, for $\alpha=\zeta \in \Gamma$ we get

$$
\left|R_{n}\left(\frac{1}{z-\zeta}\right)\right| \leq \frac{M_{n}}{|\zeta|^{q_{n}+1}(|\zeta|-1)} .
$$

Consider now a complex number $\alpha,|\alpha|<1$. Then

$$
R_{n}\left(\frac{1}{z-\alpha}\right)=R_{n}\left(\frac{1}{z} \sum_{k \geq 0}\left(\frac{\alpha}{z}\right)^{k}\right)=\sum_{k \geq 0} \alpha^{k} R_{n}\left(\frac{1}{z^{k+1}}\right)=\sum_{k \geq p_{n}} \alpha^{k} R_{n}\left(\frac{1}{z^{k+1}}\right) .
$$

Thereby

$$
\left|R_{n}\left(\frac{1}{z-\alpha}\right)\right| \leq M_{n} \sum_{k \geq p_{n}}|\alpha|^{k}=\frac{M_{n}|\alpha|^{p_{n}}}{1-|\alpha|}, z \in T,|\alpha|<1 .
$$

The proof follows by virtue of (9) and taking into account (11), (12), (14), (15) and (17). 
For the particular case $\Gamma=C_{\rho}$ where

$$
C_{\rho}=\{\zeta \in \mathbb{C}:|\zeta|=\rho\}, \rho>1
$$

we deduce the following

Corollary 1. Under the conditions of Theorem 4 with $\Gamma=C_{\rho}$ it holds

$$
\begin{aligned}
\left|R_{n}(f)\right| \leq M_{n} \rho[ & \frac{1}{|\rho-| \alpha_{1}|| \cdots|\rho-| \alpha_{\nu}|| \rho^{q_{n}+1}(\rho-1)} \\
& +\sum_{\left|\alpha_{j}\right|<1} \frac{\left|\alpha_{j}\right| p_{n}}{\left(\rho-\left|\alpha_{j}\right|\right)\left|P^{\prime}\left(\alpha_{j}\right)\right|\left(1-\left|\alpha_{j}\right|\right)} \\
& \left.+\sum_{\left|\alpha_{j}\right|>1} \frac{1}{|\rho-| \alpha_{j}||\left|P^{\prime}\left(\alpha_{j}\right)\right|\left(\left|\alpha_{j}\right|-1\right)\left|\alpha_{j}\right| q_{n}+1}\right] \max _{\zeta \in C_{\rho}}|g(\zeta)| .
\end{aligned}
$$

For a function $f$ analytic in $D$ we can also make use of Theorem 4 This is what we do in the following corollary.

Corollary 2. Let $f$ be a function analytic in D, i.e., the multiplicities $\tau_{j}$ in (5) are equal to zero for $1 \leq j \leq \nu$. Let $I_{n}$ be a quadrature formula of interpolatory type in $\Lambda_{-p_{n}, q_{n}}$, where $p_{n}$ and $q_{n}$ are nonnegative integers satisfying $p_{n}+q_{n}=n-1$. Assume that its corresponding error term satisfies (4). Let $\Gamma$ be a positively oriented Jordan curve in $D$ that contains $T$ in its interior and $0 \in D$. Then

$$
\left|R_{n}(f)\right| \leq \frac{M_{n} \ell(\Gamma)}{2 \pi b^{q_{n}+2}(b-1)} \max _{\zeta \in \Gamma}|f(\zeta)| .
$$

For the particular case $\Gamma=C_{\rho}$ we deduce

$$
\left|R_{n}(f)\right| \leq \frac{M_{n}}{\rho^{q_{n}+1}(\rho-1)} \max _{\zeta \in C_{\rho}}|f(\zeta)| .
$$

Proof. Define $h(z)=g(z) / z$ if $z \neq 0$ where $g(z)=z f(z)$ and $h(0)=f(0)$. Since $h(z)=f(z), z \in T$ and the nodes of the quadrature formula are located on $T$, it holds $R_{n}(h)=R_{n}(f)$. The proof follows by making use of Theorem 4 for the function $h(z)$.

The error bound (20) was also deduced in [8].

We consider next the general case of multiple poles, that is, we deal with functions $f$ of the form (5) with the multiplicities $\tau_{j} \geq 1$. For $\zeta \in \Gamma$ and $z \in T$ it holds that

$$
\frac{1}{(\zeta-z)\left(z-\alpha_{1}\right)^{\tau_{1}} \cdots\left(z-\alpha_{\nu}\right)^{\tau_{\nu}}}=\frac{B(\zeta)}{\zeta-z}+\sum_{j=1}^{\nu} \sum_{\ell=1}^{\tau_{j}} \frac{B_{j, \ell}(\zeta)}{\left(z-\alpha_{j}\right)^{\ell}}
$$

where

$$
\begin{aligned}
& B(\zeta)=\frac{1}{\left(\zeta-\alpha_{1}\right)^{\tau_{1}} \cdots\left(\zeta-\alpha_{\nu}\right)^{\tau_{\nu}}} \\
& B_{j, \ell}(\zeta)=\frac{1}{\left(\tau_{j}-\ell\right) !} \lim _{z \rightarrow \alpha_{j}} \frac{d^{\tau_{j}-\ell}}{d z^{\tau_{j}-\ell}}\left[\frac{\left(z-\alpha_{j}\right)^{\tau_{j}}}{(\zeta-z)\left(z-\alpha_{1}\right)^{\tau_{1}} \cdots\left(z-\alpha_{\nu}\right)^{\tau_{\nu}}}\right]
\end{aligned}
$$


for $1 \leq \ell \leq \tau_{j}, 1 \leq j \leq \nu$. Thus

$$
\begin{aligned}
K_{n}(\zeta)= & B(\zeta) R_{n}\left(\frac{1}{\zeta-z}\right)+\sum_{\left|\alpha_{j}\right|<1} \sum_{\ell=1}^{\tau_{j}} B_{j, \ell}(\zeta) R_{n}\left(\frac{1}{\left(z-\alpha_{j}\right)^{\ell}}\right) \\
& +\sum_{\left|\alpha_{j}\right|>1} \sum_{\ell=1}^{\tau_{j}} B_{j, \ell}(\zeta) R_{n}\left(\frac{1}{\left(z-\alpha_{j}\right)^{\ell}}\right), \zeta \in \Gamma, z \in T,
\end{aligned}
$$

where $B(\zeta)$ and $B_{j, \ell}(\zeta)$ are given by (21). Our goal now is to get a bound for $\max _{\zeta \in \Gamma}\left|K_{n}(\zeta)\right|$. Note that we can write

$$
B_{j, \ell}(\zeta)=\frac{C_{j, \ell}}{\left(\zeta-\alpha_{j}\right)^{\tau_{j}-\ell+1}}, 1 \leq \ell \leq \tau_{j}, 1 \leq j \leq \nu,
$$

where $C_{j, \ell}$ is a constant independent of $\zeta$. Thus

$$
|B(\zeta)| \leq \frac{1}{e},\left|B_{j, \ell}(\zeta)\right| \leq \frac{\left|C_{j, \ell}\right|}{e_{j}^{\tau_{j}-\ell+1}}, \zeta \in \Gamma,
$$

where $e_{j}, 1 \leq j \leq \nu$ is given by (10) and

$$
e=\min _{\zeta \in \Gamma}\left|\left(\zeta-\alpha_{1}\right)^{\tau_{1}} \cdots\left(\zeta-\alpha_{\nu}\right)^{\tau_{\nu}}\right| .
$$

By induction on $\ell$ it is simple to deduce the relation

$$
R_{n}\left(\frac{1}{(z-\alpha)^{\ell}}\right)=\frac{1}{(\ell-1) !} \frac{d^{\ell-1}}{d \alpha^{\ell-1}} R_{n}\left(\frac{1}{z-\alpha}\right), \ell \geq 1, z \in T, \alpha \in \mathbb{C}-T .
$$

Let us consider a complex number $\alpha$ in the open unit disc, i.e., $\alpha \in \mathbb{C},|\alpha|<1$. Taking into account (16), we can deduce from (26) that

$$
\left|R_{n}\left(\frac{1}{(z-\alpha)^{\ell}}\right)\right| \leq \frac{M_{n}}{(\ell-1) !} \sum_{k \geq k\left(p_{n}, \ell\right)} k(k-1) \cdots(k-(\ell-2))|\alpha|^{k-\ell+1}, \ell \geq 2,
$$

where $k\left(p_{n}, \ell\right)=\max \left\{p_{n}, \ell-1\right\}$.

Define for $\ell \in \mathbb{N}, \ell \geq 1, m \geq 0, m \in \mathbb{N}$ and $\alpha$ a complex number $|\alpha|<1$,

$$
S_{\alpha, m}^{(\ell)}=\sum_{k \geq k(m, \ell)} k(k-1) \cdots(k-(\ell-2))|\alpha|^{k-\ell+1}, \ell \geq 2,
$$

and for $\ell=1$

$$
S_{\alpha, m}^{(1)}=\sum_{k \geq m}|\alpha|^{k}=\frac{|\alpha|^{m}}{1-|\alpha|},
$$

where as usual $k(m, \ell)=\max \{m, \ell-1\}$.

Thus for a pole $\alpha_{j}$ of $f,\left|\alpha_{j}\right|<1$, it can be written

$$
\left|R_{n}\left(\frac{1}{\left(z-\alpha_{j}\right)^{\ell}}\right)\right| \leq \frac{M_{n}}{(\ell-1) !} S_{\alpha_{j}, p_{n}}^{(\ell)}, 1 \leq \ell \leq \tau_{j}, z \in T .
$$

Note that by virtue of (17) the above equation is also valid for $\ell=1$. Observe that $S_{\alpha, m}^{(\ell)}=\left[\frac{d^{\ell-1}}{d x^{\ell-1}}\left(\frac{x^{k(m, \ell)}}{1-x}\right)\right]_{x=|\alpha|}, \ell \in \mathbb{N}, \ell \geq 1, m \in \mathbb{N}, m \geq 0, \alpha \in \mathbb{C},|\alpha|<1$.

Furthermore, the value $S_{\alpha, m}^{(\ell)}$ can be obtained recursively according to the rule stated in the next theorem. 
Theorem 5. Let $\alpha \in \mathbb{C},|\alpha|<1, m \geq 0, m \in \mathbb{N}$ and $\ell \geq 2$, $\ell \in \mathbb{N}$. If $m \geq \ell-1$, then

$$
S_{\alpha, m}^{(\ell)}=\frac{m(m-1) \cdots(m-(\ell-2))|\alpha|^{m-\ell+1}}{1-|\alpha|}+(\ell-1) \frac{S_{\alpha, m}^{(\ell-1)}}{1-|\alpha|}, \ell \geq 2 .
$$

If $\ell-1>m$, then

$$
S_{\alpha, m}^{(\ell)}=\frac{\ell-1}{1-|\alpha|} S_{\alpha, m}^{(\ell-1)}, \ell \geq 2
$$

Proof. Consider the parial sums of $S_{\alpha, m}^{(\ell)}$ given by

$$
S_{\alpha, m, t}^{(\ell)}=\sum_{k=k(m, \ell)}^{k(m, \ell)+t-1} k(k-1) \cdots(k-(\ell-2))|\alpha|^{k-\ell+1}, t \geq 1 .
$$

The difference $S_{\alpha, m, t}^{(\ell)}-|\alpha| S_{\alpha, m, t}^{(\ell)}$ yields for $t \geq 2$

$$
\begin{aligned}
(1-|\alpha|) S_{\alpha, m, t}^{(\ell)}= & k(m, \ell)(k(m, \ell)-1) \cdots(k(m, \ell)-(\ell-2))|\alpha|^{k(m, \ell)-\ell+1} \\
& +(\ell-1) A(k(m, \ell), \alpha, \ell, t) \\
& -(k(m, \ell)+t-1) \cdots(k(m, \ell)+t-1-(\ell-2))|\alpha|^{k(m, \ell)+t-\ell+1},
\end{aligned}
$$

where

$$
\begin{aligned}
A(k(m, \ell), \alpha, \ell, t)= & k(m, \ell)(k(m, \ell)-1) \cdots(k(m, \ell)-(\ell-3))|\alpha|^{k(m, \ell)-\ell+2}+\cdots \\
& +(k(m, \ell)+t-2) \cdots(k(m, \ell)+t-2-(\ell-3))|\alpha|^{k(m, \ell)+t-\ell} .
\end{aligned}
$$

If $m \geq \ell-1$, then $k(m, \ell)=k(m, \ell-1)=m$ and hence $A(k(m, \ell), \alpha, \ell, t)=S_{\alpha, m, t-1}^{(\ell-1)}$. If $\ell-1>m$, then $k(m, \ell)=\ell-1=k(m, \ell-1)+1$ and hence

$$
\begin{aligned}
A(k(m, \ell), \alpha, \ell, t) & =S_{\alpha, m, t-1}^{(\ell-1)}-(\ell-2) ! \\
& +(k(m, \ell)+t-2) \cdots(k(m, \ell)+t-2-(\ell-3))|\alpha|^{k(m, \ell)+t-\ell} .
\end{aligned}
$$

Note that

$$
\begin{aligned}
0 & \leq \lim _{t \rightarrow \infty}(k(m, \ell)+t-1) \cdots(k(m, \ell)+t-1-(\ell-2))|\alpha|^{k(m, \ell)+t-\ell+1} \\
& \leq \lim _{t \rightarrow \infty}(2 t-1)^{\ell-1}|\alpha|^{t-\ell+1}=0 .
\end{aligned}
$$

Furthermore

$$
\begin{aligned}
0 & \leq \lim _{t \rightarrow \infty}(k(m, \ell)+t-2) \cdots(k(m, \ell)+t-2-(\ell-3))|\alpha|^{k(m, \ell)+t-\ell} \\
& \leq \lim _{t \rightarrow \infty}(2 t-2)^{\ell-2}|\alpha|^{t-\ell}=0 .
\end{aligned}
$$

The proof follows taking limit when $t$ tends to infinity in (29). 
We consider next a pole $\alpha_{j}$ of $f,\left|\alpha_{j}\right|>1$. From (26) taking into account (13) one gets

$$
\left|R_{n}\left(\frac{1}{\left(z-\alpha_{j}\right)^{\ell}}\right)\right| \leq \frac{M_{n}}{(\ell-1) !} \sum_{k \geq q_{n}+1}(k+1)(k+2) \cdots(k+\ell-1) \frac{1}{\left|\alpha_{j}\right|^{k+\ell}}, \ell \geq 2 .
$$

Replace in the last summation $k$ by $t=k+\ell-1$. We obtain

$$
\left|R_{n}\left(\frac{1}{\left(z-\alpha_{j}\right)^{\ell}}\right)\right| \leq \frac{M_{n}}{(\ell-1) !\left|\alpha_{j}\right|^{\ell}} S_{1 / \alpha_{j}, q_{n}+\ell}^{(\ell)},\left|\alpha_{j}\right|>1,1 \leq \ell \leq \tau_{j}, z \in T .
$$

Note that this equation is also valid for $\ell=1$ by virtue of (14).

We can now summarize in the following theorem.

Theorem 6. Let $I_{n}$ be a quadrature formula of interpolatory type in $\Lambda_{-p_{n}, q_{n}}$ with property (4) where $p_{n}$ and $q_{n}$ are nonnegative integers satisfying $p_{n}+q_{n}=n-1, n \geq$ 1. Let $f$ be a function of the form (5) and analytic in a simply connected domain $D$ containing $T$ in its interior. Consider a positively oriented Jordan curve $\Gamma$ in $D$ that contains $T$ in its interior and that $\Gamma \cap\left\{\alpha_{1}, \ldots, \alpha_{\nu}\right\}=\emptyset$. Then

$$
\begin{aligned}
\left|R_{n}(f)\right| \leq \frac{M_{n} \ell(\Gamma)}{2 \pi}\left[\frac{1}{e b^{q_{n}+1}(b-1)}\right. & +\sum_{\left|\alpha_{j}\right|<1} \sum_{\ell=1}^{\tau_{j}} \frac{\left|C_{j, \ell}\right| S_{\alpha_{j}, p_{n}}^{(\ell)}}{e_{j}^{\tau_{j}-\ell+1}(\ell-1) !} \\
& \left.+\sum_{\left|\alpha_{j}\right|>1} \sum_{\ell=1}^{\tau_{j}} \frac{\left|C_{j, \ell}\right| S_{1 / \alpha_{j}, q_{n}+\ell}^{(\ell)}}{e_{j}^{\tau_{j}-\ell+1}(\ell-1) !\left|\alpha_{j}\right|^{\ell}}\right] \max _{\zeta \in \Gamma}|g(\zeta)|,
\end{aligned}
$$

where $\ell(\Gamma)$ denotes the length of $\Gamma$ and $b$ and $e_{j}$ are given by (10). $C_{j, \ell}$ and $e$ are given by (23) and (25), respectively. The function $S_{\alpha, m}^{(\ell)}$ is defined in (27) and can be evaluated by means of Theorem 5 .

Proof. Take into account (91) jointly with (22), (15), (28) and (30).

In the case of simple poles, i.e., $\tau_{j}=1,1 \leq j \leq \nu$, Theorem 6 reduces to Theorem 4

Corollary 3. Under the conditions of Theorem 6 it holds for $\Gamma=C_{\rho}=\{\zeta \in \mathbb{C}$ : $|\zeta|=\rho\}, \rho>1$ that

$$
\begin{aligned}
\left|R_{n}(f)\right| \leq M_{n} \rho[ & \frac{1}{|\rho-| \alpha_{1}\left|\tau^{\tau_{1}} \cdots\right| \rho-\left.\left|\alpha_{\nu}\right|\right|^{\tau_{\nu}} \rho^{q_{n}+1}(\rho-1)} \\
& +\sum_{\left|\alpha_{j}\right|<1} \sum_{\ell=1}^{\tau_{j}} \frac{\left|C_{j, \ell}\right| S_{\alpha_{j}, p_{n}}^{(\ell)}}{\left(\rho-\left|\alpha_{j}\right|\right)^{\tau_{j}-\ell+1}(\ell-1) !} \\
& \left.+\sum_{\left|\alpha_{j}\right|>1} \sum_{\ell=1}^{\tau_{j}} \frac{\left|C_{j, \ell}\right| S_{1 / \alpha_{j}, q_{n}+\ell}^{(\ell)}}{|\rho-| \alpha_{j}||^{\tau_{j}-\ell+1}(\ell-1) !\left|\alpha_{j}\right|^{\ell}}\right] \max _{\zeta \in C_{\rho}}|g(\zeta)| .
\end{aligned}
$$




\section{Convergence Analysis}

As we mentioned in Theorem 2, the convergence of interpolatory rules for bounded Riemann integrable functions on $T$, based on the zeros of para-orthogonal polynomials with respect to a given distribution function, is constrained to the condition that when $n$ tends to infinity then the parameters at our disposal, $p_{n}$ and $q_{n}, p_{n}+q_{n}=n-1$, must both tend to infinity. In the case that one of the parameters is fixed to a given value and the other tends to infinity, then convergence may be lost. This case is shown in Example 1 given below where a nonsuitable fixed value leads into a nonconvergent sequence of quadrature formulas. But as we will show, for a subclass of functions of (5) we can appropriately fix a value for one of the parameters while retaining convergence. The advantage of this strategy is to minimize the quadrature error bound.

Example 1. Consider $f(z)=z$. (Note that we can write $f(z)=g(z) / z$ where $g(z)=z^{2}$.) For $n \geq 1$, take $q_{n}=0$ and hence $p_{n}=n-1$. Consider the Poisson integral

$$
I(f)=\int_{-\pi}^{\pi} f\left(e^{i \theta}\right) d \psi(\theta),
$$

where $\psi(\theta)$ is the absolutely continuous distribution function with derivative

$$
\psi^{\prime}(\theta)=\frac{1-|r|^{2}}{\left|e^{i \theta}-r\right|^{2}}, r \in \mathbb{C}, 0 \leq|r|<1,-\pi \leq \theta<\pi .
$$

It holds that

$$
I\left(z^{k}\right)=2 \pi r^{k}, I\left(\frac{1}{z^{k}}\right)=2 \pi \bar{r}^{k}, k \geq 0 .
$$

Consider any fixed $r \in \mathbb{C}, 0<|r|<1$. Let $I_{n}$ be the quadrature formula of interpolatory type in $\Lambda_{-(n-1), 0}$ to estimate the integral (32) with uniformly distributed nodes on $T$, that is, the nodes are the roots of $z^{n}+\kappa_{n}=0, \kappa_{n} \in \mathbb{C},\left|\kappa_{n}\right|=1, n \geq 1$. The L-polynomial in $\Lambda_{-(n-1), 0}$ interpolating $f$ at such a set of nodes is $-\kappa_{n} / z^{n-1}$. Then taking into account the remark after Definition 10 wet $I_{n}(f)=I_{n}(z)=$ $I\left(-\kappa_{n} / z^{n-1}\right)=-2 \pi \kappa_{n} \bar{r}^{n-1}$. Thus $\lim _{n \rightarrow \infty} I_{n}(f)=0 \neq I(f)=2 \pi r$.

On the other hand, consider $q_{n}=q, q \geq 1, n \geq q+1$ and hence $p_{n}=n-1-q$. Let $I_{n}$ be the quadrature formula of interpolatory type in $\Lambda_{-p_{n}, q}$ with uniformly distributed nodes on $T$ to estimate (32). Then $I_{n}(f)$ converges to $I(f)=I(z)$ since by construction $I_{n}(f)=I(f), n \geq q+1$.

From here on we will assume that the constant $M_{n}$ in (4) is also independent of $n$, so we will write $M$ rather than $M_{n}$. As we saw in Section 1 , this is the case of the most frecuently used quadrature formulas on the unit circle.

Suppose first that all the poles lie inside the open unit disc, that is, $\left|\alpha_{j}\right|<1,1 \leq$ $j \leq \nu$. Let $\alpha$ be a pole with maximum modulus. From (31) we can write

$$
\begin{aligned}
\left|R_{n}(f)\right| \leq M \rho & {\left[\frac{1}{(\rho-|\alpha|)^{\tau} \rho^{q_{n}+1}(\rho-1)}\right.} \\
& \left.+\sum_{j=1}^{\nu} \sum_{\ell=1}^{\tau_{j}} \frac{\left|C_{j, \ell}\right| S_{\alpha_{j}, p_{n}}^{(\ell)}}{\left(\rho-\left|\alpha_{j}\right|\right)^{\tau_{j}-\ell+1}(\ell-1) !}\right] \max _{\zeta \in C_{\rho}}|g(\zeta)|,
\end{aligned}
$$


where $\tau=\tau_{1}+\cdots+\tau_{\nu}$. From definition (27) of $S_{\alpha, m}^{(\ell)}$ we deduce that

$$
S_{\alpha_{j}, p_{n}}^{(\ell)} \leq \sum_{k \geq p_{n}} k^{\hat{\tau}-1}|\alpha|^{k-\hat{\tau}+1}<+\infty, 1 \leq j \leq \nu, 1 \leq \ell \leq \tau_{j}, p_{n}>\hat{\tau}-1,
$$

where $\hat{\tau}=\max _{1 \leq j \leq \nu} \tau_{j}$. Furthermore if $\rho-|\alpha|>1$, then

$$
\left|R_{n}(f)\right| \leq M \rho\left[\frac{1}{(\rho-|\alpha|)^{\tau} \rho^{q_{n}+1}(\rho-1)}+\frac{\tau \hat{M}}{\rho-|\alpha|} \sum_{k \geq p_{n}} k^{\hat{\tau}-1}|\alpha|^{k-\hat{\tau}+1}\right] \max _{\zeta \in C_{\rho}}|g(\zeta)|,
$$

where $\hat{M}=\max _{1 \leq j \leq \nu, 1 \leq \ell \leq \tau_{j}}\left|C_{j, \ell}\right|$.

Let us assume that $g$ is an entire function satisfying

$$
\max _{\zeta \in C_{\rho}}|g(\zeta)| \leq c \rho^{d}, \rho>1,
$$

where $c \geq 0$ and $d \geq 0, d \in \mathbb{N}$, are constants independent of $\rho$. From Liouville's theorem, see, e.g., [4, p. 159], it follows that the set of functions $g$ satisfying (36) is the set of polynomials of degree at most $d$.

Taking into account (35) and (36) we get

$$
\begin{aligned}
\left|R_{n}(f)\right| \leq M c \rho^{d+1}[ & \frac{1}{(\rho-|\alpha|)^{\tau} \rho^{q_{n}+1}(\rho-1)} \\
& \left.+\frac{\tau \hat{M}}{\rho-|\alpha|} \sum_{k \geq p_{n}} k^{\hat{\tau}-1}|\alpha|^{k-\hat{\tau}+1}\right], p_{n}>\hat{\tau}-1 .
\end{aligned}
$$

Observe that

$$
\begin{aligned}
\sum_{k \geq p_{n}} k^{\hat{\tau}-1}|\alpha|^{k-\hat{\tau}+1} & =p_{n}^{\hat{\tau}-1}|\alpha|^{p_{n}-\hat{\tau}+1} \sum_{k \geq 0}\left(\frac{p_{n}+k}{p_{n}}\right)^{\hat{\tau}-1}|\alpha|^{k} \\
& \leq p_{n}^{\hat{\tau}-1}|\alpha|^{p_{n}-\hat{\tau}+1} D_{\alpha, \hat{\tau}}
\end{aligned}
$$

where

$$
D_{\alpha, \hat{\tau}}=\sum_{k \geq 0}(1+k)^{\hat{\tau}-1}|\alpha|^{k}<+\infty .
$$

Hence

$$
\begin{aligned}
\left|R_{n}(f)\right| \leq M c \rho^{d+1}[ & \frac{1}{(\rho-|\alpha|)^{\tau} \rho^{q_{n}+1}(\rho-1)} \\
& \left.+\frac{\tau \hat{M} D_{\alpha, \hat{\tau}} p_{n}^{\hat{\tau}-1}|\alpha|^{p_{n}-\hat{\tau}+1}}{\rho-|\alpha|}\right], p_{n}>\hat{\tau}-1 .
\end{aligned}
$$

From where we deduce that if both $p_{n}$ and $q_{n}, p_{n}+q_{n}=n-1$ tend to infinity as $n$ tends to infinity we get convergence for any fixed $\rho, \rho-|\alpha|>1$. Convergence is also assured if $q_{n}$ is fixed to a nonnegative integer $q_{n}=q \geq d-\tau$ and $\lim _{n \rightarrow \infty} p_{n}=\infty$, $p_{n}+q_{n}=n-1$. Indeed, from (37)

$$
\lim _{n \rightarrow \infty}\left|R_{n}(f)\right| \leq \frac{M c \rho^{d+1}}{(\rho-|\alpha|)^{\tau} \rho^{q+1}(\rho-1)}
$$

for any $\rho$ such that $\rho-|\alpha|>1$. The assertion follows taking infimum in $\rho$. 
The right hand part of (37) as a function of $p_{n}$ is decreasing for sufficiently large $p_{n}$. Thus we suggest for functions of the form (5) with all its poles in the open unit disc and satisfying (36), that is, the rational functions with poles on the open unit disc, to take $q_{n}=q=\max \{0, d-\tau\}, \tau=\tau_{1}+\cdots+\tau_{\nu}$, and hence $p_{n}=n-1-q$.

Suppose now that all the poles lie outside the closed unit disc, that is, $\left|\alpha_{j}\right|>$ $1,1 \leq j \leq \nu$. Then from

$$
\begin{aligned}
\left|R_{n}(f)\right| \leq M \rho & {\left[\frac{1}{|\rho-| \beta||^{\tau} \rho^{q_{n}+1}(\rho-1)}\right.} \\
& \left.+\sum_{j=1}^{\nu} \sum_{\ell=1}^{\tau_{j}} \frac{\left|C_{j, \ell}\right| S_{1 / \alpha_{j}, q_{n}+\ell}^{(\ell)}}{|\rho-| \alpha_{j}||^{\tau_{j}-\ell+1}(\ell-1) !\left|\alpha_{j}\right|^{\ell}}\right] \max _{\zeta \in C_{\rho}}|g(\zeta)|,
\end{aligned}
$$

where $\beta$ is a pole for which is attained $\min _{\alpha_{j}}|\rho-| \alpha_{j}||$ and as usual $\tau=\tau_{1}+\cdots+\tau_{\nu}$. From the definition (27) of $S_{\alpha, m}^{(\ell)}$ we deduce that

$$
S_{1 / \alpha_{j}, q_{n}+\ell}^{(\ell)} \leq \sum_{k \geq q_{n}} k^{\hat{\tau}-1}|\hat{\beta}|^{k-\hat{\tau}+1}<\infty, 1 \leq j \leq \nu, 1 \leq \ell \leq \tau_{j}, q_{n}>\hat{\tau}-1
$$

where $\hat{\beta}=\max _{1 \leq j \leq \nu} 1 /\left|\alpha_{j}\right|$.

Then

$$
\begin{aligned}
\left|R_{n}(f)\right| \leq M \rho & {\left[\frac{1}{|\rho-| \beta||^{\tau} \rho^{q_{n}+1}(\rho-1)}\right.} \\
& \left.+\frac{\tau \hat{B}}{\left.|\rho-| \beta\right|^{\delta}} \sum_{k \geq q_{n}} k^{\hat{\tau}-1}|\hat{\beta}|^{k-\hat{\tau}+1}\right] \max _{\zeta \in C_{\rho}}|g(\zeta)|,
\end{aligned}
$$

where $\hat{B}=\max _{1 \leq j \leq \nu, 1 \leq \ell \leq \tau_{j}}\left|C_{j, \ell}\right| /\left((\ell-1) !\left|\alpha_{j}\right|^{\ell}\right), \quad \hat{\tau}=\max _{1 \leq j \leq \nu} \tau_{j}, \delta=1$ if $|\rho-| \beta|| \geq 1$ and $\delta=\hat{\tau}$ if $|\rho-| \beta||<1$.

The expression in brackets in the right hand part of (38) decreases for increasing values of $q_{n}, 0 \leq q_{n} \leq n-1, n$ sufficiently large. Hence for functions of the form (51) with all its poles outside the closed unit disc we suggest $q_{n}=n-1$ and hence $p_{n}=0, n \geq 1$. Note that this choice also assures convergence.

If the function $f$ is analytic, then the bound (20) attains its minimum for $q_{n}=$ $n-1$ and hence $p_{n}=0, n \geq 1$. We also find convergence (if $M_{n}$ does not depend on $n$ ).

If there are poles of $f$ in the interior and exterior of the open unit disc, then one can decompose $1 /\left(z-\alpha_{1}\right)^{\tau_{1}} \cdots\left(z-\alpha_{\nu}\right)^{\tau_{\nu}}$ into two parts: the first part, say $h_{1}$, with the singularities in the open unit disc and the second part, $h_{2}$, with all the singularities located in the exterior of the closed unit disc. Then $I(f)=I\left(g h_{1}\right)+$ $I\left(g h_{2}\right)$. Now we can approximate both integrals by means of two interpolatory type quadrature rules with the values of $p_{n}$ and $q_{n}$ previously proposed to each case. 


\section{NumERICAL EXAMPLES}

In this section we show by means of several numerical examples the effectiveness of our guidelines for the choice of the parameters $p_{n}$ and $q_{n}$. For the numerical examples we consider quadrature formulas

$$
I_{n}(f)=\sum_{j=1}^{n} c_{j, n} f\left(z_{j, n}\right) \doteq \int_{-\pi}^{\pi} f\left(e^{i \theta}\right) d \psi(\theta)
$$

of interpolatory type in $\Lambda_{-p_{n}, q_{n}}, p_{n}$ and $q_{n}$ nonnegative integers such that $p_{n}+q_{n}=$ $n-1$, with uniformly distributed nodes $z_{j, n}, 1 \leq j \leq n$ on $T$, i.e., the nodes are the roots of $z^{n}+\kappa_{n}=0, \kappa_{n} \in \mathbb{C},\left|\kappa_{n}\right|=1, n \geq 1$. We will take $\kappa_{n}=-1, n \geq 1$ in all the examples.

We will consider the absolutely continuous distribution function $\psi$ given by

$$
\psi(\theta)=\int_{-\pi}^{\theta} \psi^{\prime}(t) d t,-\pi \leq \theta<\pi,
$$

where $\psi^{\prime}$ is given by (33).

The coefficients $c_{j, n}, 1 \leq j \leq n, n \geq 1$ are given by ([8])

$$
c_{j, n}=\frac{1}{n} \sum_{\ell=-p_{n}}^{q_{n}} m_{\ell} \frac{w^{(1-j) \ell}}{z_{1, n}^{\ell}}, w=e^{2 \pi i / n}, m_{\ell}=I\left(z^{\ell}\right) .
$$

Since we have fixed $\kappa_{n}=-1, n \geq 1$, we take $z_{1, n}=1$ for $n \geq 1$. Taking into account (39) and (34) we obtain

$$
c_{j, n}=\frac{2 \pi}{n}\left[\sum_{\ell=-1}^{-p_{n}} \bar{r}^{|\ell|} w^{(1-j) \ell}+\sum_{\ell=0}^{q_{n}} r^{\ell} w^{(1-j) \ell}\right] .
$$

Each term in brackets is a geometric sum. Thus

$$
c_{j, n}=\frac{2 \pi}{n}\left[\frac{w^{(1-j)\left(p_{n}+1\right)}-\bar{r}^{p_{n}+1}}{\left(w^{1-j}-\bar{r}\right) w^{(1-j) p_{n}}}-1+\frac{1-\left(r w^{1-j}\right)^{q_{n}+1}}{1-r w^{1-j}}\right], 1 \leq j \leq n, n \geq 1 .
$$

In the following examples, all the tables list the absolute error $\left|R_{n}(f)\right|$ achieved. We have taken $r=0.5$ in all the examples.

Example 2. Consider $f(z)=g(z) /(z-0.2)^{2}$ where $g(z)=z^{3}$. One has $I(f)=$ $157 \pi / 81$. The function $f$ has a pole of order two at $z=0.2$ in the open unit disc. Thus the sum of the multiplicities of the poles is $\tau=2$. The function $g(z)$ satisfies $\max _{\zeta \in C_{\rho}}|g(z)| \leq c \rho^{d}, \rho>1$ with $c=1$ and $d=3$. Thereby $q_{n}=q=$ $\max \{0, d-\tau\}=\max \{0,1\}=1$ and hence $p_{n}=n-2$ minimize the error bound (37) from a certain $n$ on. See Table 1 .

Example 3. Let $f(z)=g(z) /(z-2)$ where $g(z)=z^{3}$. It holds $I(f)=-\pi / 6$. The function $f$ has a simple pole at $z=2$ in the exterior of the closed unit disc. Thus for $p_{n}=0$ and hence $q_{n}=n-1$ the error bound (38) attains its minimum from a certain $n$ on. See Table 2.

Example 4. We consider here the case of an analytic function. Let $f(z)=e^{z}$. One has $I(f)=2 \pi e^{1 / 2}$. The values $q_{n}=n-1$ and hence $p_{n}=0, n \geq 1$, were proposed for analytic functions. See Table 3. 
Example 5. Let $f(z)=g(z) /((z-\alpha)(z-\beta))$ where $g(z)=z^{3}, \alpha=0.25$ and $\beta=3$. One finds $I(f)=-4 \pi(-3 / 313+1 / 10)$. We have compared two procedures. For the first one, calculate $I_{n}(f)$ as interpolatory type in $\Lambda_{-p_{n}, q_{n}}$. In this case, the error listed and the integer in brackets behind it are the lesser error and the value of $q_{n}$ for which this error is attained respectively. This value of $q_{n}$ is not known in advance, so we have taken it from the numerical results. In the second procedure, which we denote by PFD, we calculate the partial fraction decomposition of $1 /(z-\alpha)(z-\beta)=$ $-a z^{3} /(z-\alpha)+a z^{3} /(z-\beta), a=4 / 11$ and then $I(f)=I\left(-a z^{3} /(z-\alpha)\right)+$ $I\left(a z^{3} /(z-\beta)\right)$. Taking into account our guidelines, we approximate the integral $I\left(-a z^{3} /(z-\alpha)\right)$ by means of the quadrature formula of interpolatory type in $\Lambda_{-p_{n}, q_{n}}=\Lambda_{-(n-3), 2}$, and for the integral $I\left(a z^{3} /(z-\beta)\right)$, we take $p_{n}=0$ and hence $q_{n}=n-1, n \geq 1$. See Table 4 .

TABLE 1.

\begin{tabular}{rlllll}
\hline$q_{n}$ & $n=4$ & $n=6$ & $n=8$ & $n=10$ & $n=12$ \\
\hline 0 & $.234 \mathrm{D}+01$ & $.294 \mathrm{D}+01$ & $.309 \mathrm{D}+01$ & $.313 \mathrm{D}+01$ & $.314 \mathrm{D}+01$ \\
1 & $.317 \mathrm{D}-01$ & $.203 \mathrm{D}-02$ & $.107 \mathrm{D}-03$ & $.525 \mathrm{D}-05$ & $.248 \mathrm{D}-06$ \\
2 & $.317 \mathrm{D}-01$ & $.430 \mathrm{D}-02$ & $.258 \mathrm{D}-03$ & $.132 \mathrm{D}-04$ & $.632 \mathrm{D}-06$ \\
3 & $.252 \mathrm{D}+00$ & $.430 \mathrm{D}-02$ & $.522 \mathrm{D}-03$ & $.301 \mathrm{D}-04$ & $.150 \mathrm{D}-05$ \\
4 & & $.334 \mathrm{D}-01$ & $.522 \mathrm{D}-03$ & $.603 \mathrm{D}-04$ & $.339 \mathrm{D}-05$ \\
5 & & $.387 \mathrm{D}+00$ & $.419 \mathrm{D}-02$ & $.603 \mathrm{D}-04$ & $.678 \mathrm{D}-05$ \\
6 & & & $.513 \mathrm{D}-01$ & $.505 \mathrm{D}-03$ & $.678 \mathrm{D}-05$ \\
7 & & & $.422 \mathrm{D}+00$ & $.640 \mathrm{D}-02$ & $.592 \mathrm{D}-04$ \\
8 & & & & $.559 \mathrm{D}-01$ & $.766 \mathrm{D}-03$ \\
9 & & & & $.431 \mathrm{D}+00$ & $.695 \mathrm{D}-02$ \\
10 & & & & & $.570 \mathrm{D}-01$ \\
11 & & & & & $.434 \mathrm{D}+00$ \\
\hline
\end{tabular}

TABLE 2 .

\begin{tabular}{rlllll}
\hline$q_{n}$ & $n=8$ & $n=10$ & $n=12$ & $n=14$ & $n=16$ \\
\hline 0 & $.685 \mathrm{D}-01$ & $.327 \mathrm{D}+00$ & $.462 \mathrm{D}+00$ & $.505 \mathrm{D}+00$ & $.518 \mathrm{D}+00$ \\
1 & $.928 \mathrm{D}-01$ & $.321 \mathrm{D}+00$ & $.461 \mathrm{D}+00$ & $.505 \mathrm{D}+00$ & $.518 \mathrm{D}+00$ \\
2 & $.986 \mathrm{D}-01$ & $.319 \mathrm{D}+00$ & $.460 \mathrm{D}+00$ & $.505 \mathrm{D}+00$ & $.518 \mathrm{D}+00$ \\
3 & $.394 \mathrm{D}+00$ & $.491 \mathrm{D}-01$ & $.736 \mathrm{D}-01$ & $.114 \mathrm{D}+00$ & $.126 \mathrm{D}+00$ \\
4 & $.394 \mathrm{D}+00$ & $.123 \mathrm{D}+00$ & $.184 \mathrm{D}-01$ & $.169 \mathrm{D}-01$ & $.280 \mathrm{D}-01$ \\
5 & $.320 \mathrm{D}+00$ & $.123 \mathrm{D}+00$ & $.368 \mathrm{D}-01$ & $.614 \mathrm{D}-02$ & $.384 \mathrm{D}-02$ \\
6 & $.228 \mathrm{D}+00$ & $.104 \mathrm{D}+00$ & $.368 \mathrm{D}-01$ & $.107 \mathrm{D}-01$ & $.192 \mathrm{D}-02$ \\
7 & $.131 \mathrm{D}+00$ & $.814 \mathrm{D}-01$ & $.322 \mathrm{D}-01$ & $.107 \mathrm{D}-01$ & $.307 \mathrm{D}-02$ \\
8 & & $.572 \mathrm{D}-01$ & $.265 \mathrm{D}-01$ & $.959 \mathrm{D}-02$ & $.307 \mathrm{D}-02$ \\
9 & & $.327 \mathrm{D}-01$ & $.204 \mathrm{D}-01$ & $.815 \mathrm{D}-02$ & $.278 \mathrm{D}-02$ \\
10 & & & $.143 \mathrm{D}-01$ & $.664 \mathrm{D}-02$ & $.242 \mathrm{D}-02$ \\
11 & & & $.818 \mathrm{D}-02$ & $.511 \mathrm{D}-02$ & $.204 \mathrm{D}-02$ \\
12 & & & & $.358 \mathrm{D}-02$ & $.166 \mathrm{D}-02$ \\
13 & & & & $.205 \mathrm{D}-02$ & $.128 \mathrm{D}-02$ \\
14 & & & & & $.895 \mathrm{D}-03$ \\
15 & & & & & $.511 \mathrm{D}-03$ \\
\hline
\end{tabular}


TABLE 3.

\begin{tabular}{rlllll}
\hline$q_{n}$ & $n=4$ & $n=6$ & $n=8$ & $n=10$ & $n=12$ \\
\hline 0 & $.171 \mathrm{D}+01$ & $.345 \mathrm{D}+01$ & $.392 \mathrm{D}+01$ & $.404 \mathrm{D}+01$ & $.407 \mathrm{D}+01$ \\
1 & $.666 \mathrm{D}+00$ & $.506 \mathrm{D}+00$ & $.827 \mathrm{D}+00$ & $.908 \mathrm{D}+00$ & $.928 \mathrm{D}+00$ \\
2 & $.666 \mathrm{D}+00$ & $.829 \mathrm{D}-01$ & $.904 \mathrm{D}-01$ & $.134 \mathrm{D}+00$ & $.145 \mathrm{D}+00$ \\
3 & $.272 \mathrm{D}+00$ & $.829 \mathrm{D}-01$ & $.773 \mathrm{D}-02$ & $.117 \mathrm{D}-01$ & $.165 \mathrm{D}-01$ \\
4 & & $.338 \mathrm{D}-01$ & $.773 \mathrm{D}-02$ & $.604 \mathrm{D}-03$ & $.119 \mathrm{D}-02$ \\
5 & & $.924 \mathrm{D}-02$ & $.282 \mathrm{D}-02$ & $.604 \mathrm{D}-03$ & $.410 \mathrm{D}-04$ \\
6 & & & $.778 \mathrm{D}-03$ & $.195 \mathrm{D}-03$ & $.410 \mathrm{D}-04$ \\
7 & & & $.164 \mathrm{D}-03$ & $.488 \mathrm{D}-04$ & $.118 \mathrm{D}-04$ \\
8 & & & & $.104 \mathrm{D}-04$ & $.265 \mathrm{D}-05$ \\
9 & & & & $.181 \mathrm{D}-05$ & $.523 \mathrm{D}-06$ \\
10 & & & & & $.923 \mathrm{D}-07$ \\
11 & & & & & $.136 \mathrm{D}-07$ \\
\hline
\end{tabular}

TABLE 4.

\begin{tabular}{rlllll}
\hline & $n=6$ & $n=12$ & $n=18$ & $n=24$ & $n=30$ \\
\hline$I_{n}(f)$ & $.152 \mathrm{D}-01(5)$ & $.119 \mathrm{D}-03(9)$ & $.506 \mathrm{D}-06(12)$ & $.428 \mathrm{D}-08(15)$ & $.178 \mathrm{D}-11(19)$ \\
$\mathrm{PFD}$ & $.336 \mathrm{D}-01$ & $.465 \mathrm{D}-04$ & $.637 \mathrm{D}-07$ & $.874 \mathrm{D}-10$ & $.121 \mathrm{D}-12$ \\
\hline
\end{tabular}

\section{ACKNOWLEDGMents}

The author is grateful to the referee. Their suggestions simplify the convergence analysis in Section 3. The use of derivatives to calculate $S_{\alpha, m}^{(\ell)}$, was also due to the referee.

\section{REFERENCES}

[1] A. Bultheel, P. González-Vera, E. Hendriksen and O. Njåstad, Orthogonal rational functions and interpolatory product rules on the unit circle. III: Convergence of general sequences, Preprint.

[2] A. Bultheel, P. González-Vera, E. Hendriksen and O. Njåstad, Orthogonality and quadrature on the unit circle, IMACS Annals on Computing and Appl. Math. 9 (1991) 205-210. CMP 94:10

[3] P. González-Vera, O. Njåstad and J.C. Santos-León, Some results about numerical quadrature on the unit circle, Adv. Comput. Math. 5 (1996) 297-328. MR 98f:41028

[4] P. Henrici, Applied and computational complex analysis (Vol. 1, John Wiley and Sons, New York, 1974). MR 51:8378

[5] W.B. Jones, O. Njåstad and W.J. Thron, Continued fractions associated with trigonometric and other strong moment problems, Constr. Approx. 2 (1986) 197-211. MR 88m:30087

[6] W.B. Jones, O. Njåstad and W.J. Thron, Moment theory, orthogonal polynomials, quadrature, and continued fractions associated with the unit circle, Bull. London Math. Soc. 21 (1989) 113152. MR 90e: 42027

[7] W.B. Jones and H. Waadeland, Bounds for remainder term in Szegö quadrature on the unit circle, Approximation and Computation: A. Festschrift in Honor of Walter Gautschi (Boston) (R.V.M. Zahar, ed.), International Series of Numerical Mathematics, vol. 119, Birkhäuser, Boston, 1994, pp. 325-342. MR 96i:41029

[8] J.C. Santos-León, Product rules on the unit circle with uniformly distributed nodes. Error bounds for analytic functions, J. Comput. Appl. Math. 108 (1999) 195-208. CMP 99:17

[9] G. Szegö, Orthogonal polynomials (Amer. Math. Soc. Providence, R.I., 1939).

Department of Mathematical Analysis, La Laguna University, 38271-La Laguna, Tenerife, Canary Islands, Spain

E-mail address: jcsantos@ull.es 